\title{
OPTIMIZACIÓN POR RECOCIDO SIMULADO DE UN CONVERTIDOR MULTINIVEL MONOFÁSICO CON MODULACIÓN PWM SINUSOIDAL DE MÚLTIPLE PORTADORA
}

\author{
SIMULATED ANNEALING FOR OPTIMIZATION OF A SINGLE PHASE \\ MULTILEVEL INVERTER WITH MULTIPLE CARRIER SINUSOIDAL PWM
}

\author{
MSc. José Antonio Araque*, MSc. Jorge Luis Díaz Rodríguez. ** \\ MSc. Alejandro Sallyth Guerrero.*
}

*Universidad de Sucre, Facultad de Ingeniería.

Cra. 28 \# 5-267 Barrio Puerta Roja, Sincelejo, Sucre. Tel.: (+575) 2821240.

** Universidad de Pamplona, Facultad de Ingenierías y Arquitectura, Grupo A\&C. Ciudadela Universitaria, Pamplona, Norte de Santander, Tel.: (+577) 568 5303, Ext. 164.

E-mail: \{jose.araque, alejandro.guerrero\}@unisucre.edu.co, jdiazcu@gmail.com

Resumen: Este trabajo presenta la aplicación del método heurístico de "Recocido Simulado" (Simulated Annealing) en la optimización de los parámetros de operación de un convertidor multinivel monofásico en cascada con un esquema de modulación sinusoidal de múltiple portadora (MCSPWM). El objetivo es encontrar los parámetros de modulación MCSPWM más apropiados que permitan obtener un THD por debajo del 5\% usando el menor número posible de dispositivos de conmutación en el inversor y conmutando a la frecuencia más baja posible.

Palabras clave: Recocido simulado, THD, convertidor multinivel, modulación sinusoidal de múltiple portadora

\begin{abstract}
This paper presents the application of heuristic optimization called Simulated Annealing in the optimization of the operation parameters of a cascaded single phase multilevel inverter with multiple carrier sinusoidal PWM. The aim is to find the best parameters of the multiple carrier pulse width modulation (MCSPWM) that permits obtain a total distortion harmonic factor less than $5 \%$ with the minimal number of stages in a cascaded multilevel inverter and the lowest possible frequency of commutation.
\end{abstract}

Keywords: Simulated Annealing, THD, multilevel converter, multiple carrier sinusoidal modulation.

\section{INTRODUCCIÓN}

El convertidor multinivel es un sistema de electrónica de potencia que involucra un alto número de dispositivos conmutadores basados en semiconductores que desarrollan la conversión de la energía eléctrica proporcionada por fuentes de corriente directa en pequeños pasos, logrando una forma de onda de salida de corriente alterna con bajo contenido armónico (Skvarenina, 2002).
El primer diseño de convertidor multinivel fue patentado en el año 1975 (Baker et al., 1975). En 1980 se registró el de enclavamiento de diodos (NPC) (Baker, 1980). A mediados de los años 90 fue introducida la topología por condensador volante (Lavieville et al., 1997), (Meynard et al., 1998). Esta topología ofreció varias ventajas sobre el inversor NPC (Rodriguez et al., 2002). El convertidor multinivel en cascada con fuentes de DC separadas en el año 1997 (Peng y Lai, 1997). 
Entre los trabajos más relevantes en el área de la conversión de energía eléctrica multinivel se puede citar a (Rodriguez et al., 2002) y (Pandey, et al. 2006), donde se hace una revisión exhaustiva del estado del arte de la conversión de múltiples niveles, se estudian las diferentes topologías y se exponen las aplicaciones más importantes de esta tecnología. Un trabajo muy interesante que aborda el tema de la simulación de convertidores multinivel se puede apreciar en (Malinowski et al., 2007). Otro aporte significativo lo han hecho trabajos de maestría y doctorado enfocados en los convertidores multinivel, (Rios, 2003), (Negroni, 2007), (Lega, 2007) y (Chavarría, 2010).

Entre las estrategias de modulación para hallar los ángulos de disparo de los semiconductores de potencia se encuentra la modulación MCSPWM, que consiste en la comparación de una señal sinusoidal portadora con una serie de señales de referencia triangulares. El índice de modulación y la disposición de las señales de referencia determinan la calidad de la forma de onda de salida resultante en el convertidor.

Para evaluar la calidad de la energía eléctrica suele emplearse el valor de THD o factor de distorsión armónica total, el cual es una medida del contenido armónico de la forma de onda de salida. Entre más bajo es el THD, mejor es la calidad de la señal. Debido a que en los circuitos inversores la forma de onda de salida es generada por medio de la conmutación de dispositivos semiconductores, es necesario encontrar los ángulos de disparo apropiados que permitan minimizar el THD en la salida del convertidor usando el menor número posible de dispositivos de potencia. Esto puede verse como un problema de optimización. Las técnicas de optimización heurística han surgido como una alternativa a los métodos clásicos, ya que al estar inspirados en procesos naturales resultan menos afectados por los máximos locales que las técnicas tradicionales. El algoritmo de recocido simulado (Simulated Annealing o SA) fue introducido en la década de 1980 por K. Patrick y sus colaboradores (Kirkpatrick et al., 1983), basados en ideas formuladas en los años 50. Este método simula el proceso de recocido en el cual una sustancia es calentada por encima de su punto de fusión y entonces gradualmente es enfriada para producir una estructura cristalina, la cual minimiza su probabilidad de distribución de energía. Esta estructura cristalina, compuesta de millones de átomos perfectamente alineados, es un ejemplo de la naturaleza encontrando una estructura óptima (Bertsimas y Tsitsiklis, 1993).

\section{CONVERTIDORES MULTINIVEL}

\subsection{Conversión Multinivel}

En la figura 1 se puede apreciar el diagrama esquemático de un inversor monofásico con diferente número de niveles. Aquílos dispositivos de conmutación se presentan como interruptores ideales. La generación del voltaje de salida $V a$ se logra llevando a cabo una secuencia de conmutación apropiada de las fuentes de tensión directa $V c$ que alimentan al circuito. A mayor número de fuentes, se obtiene un mayor número de niveles de salida en el convertidor

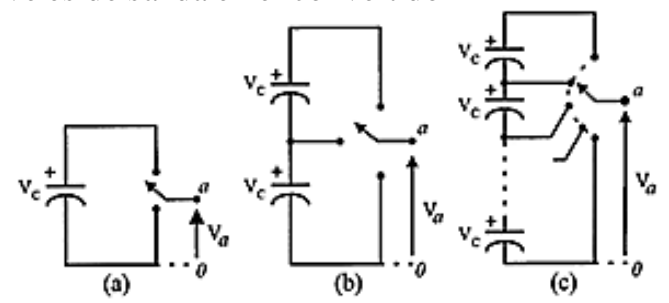

Fig. 1. Inversor monofásico.

(a) dos niveles, (b) tres niveles, $(c) m$ niveles.

Como se puede observar en la figura 2 , en un convertidor multinivel se busca sintetizar una forma de onda mucho más parecida a una señal sinusoidal, en la cual, dependiendo del número de fuentes de DC disponibles, la distorsión va a ser mucho más baja. Entre sus principales ventajas podemos destacar (Rodriguez et al., 2002):

- La disposición del voltaje de entrada en múltiples niveles permite aumentar varias veces la tensión de trabajo del convertidor empleando los mismos interruptores que un convertidor convencional.

- La potencia de los convertidores se incrementa al emplear voltajes mayores, sin necesidad de incrementar la corriente, evitando así mayores pérdidas durante la conducción, y por consecuencia mejorar el rendimiento del convertidor.

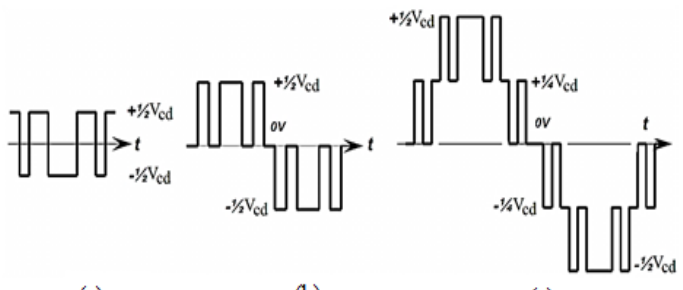

(a)

(b)

(c)

Fig. 2. Salida de voltaje del inversor (a) 2 niveles, (b) 3 niveles, (c) 5 niveles. 


\subsection{Invers or multinivel en cascada}

Esta topología se basa en la conexión en serie de inversores monofásicos tipo puente $\mathrm{H}$ con fuentes de alimentación independientes.

En la Fig. 3 se muestra el circuito de la sección de potencia para un inversor monofásico de $N$ etapas:

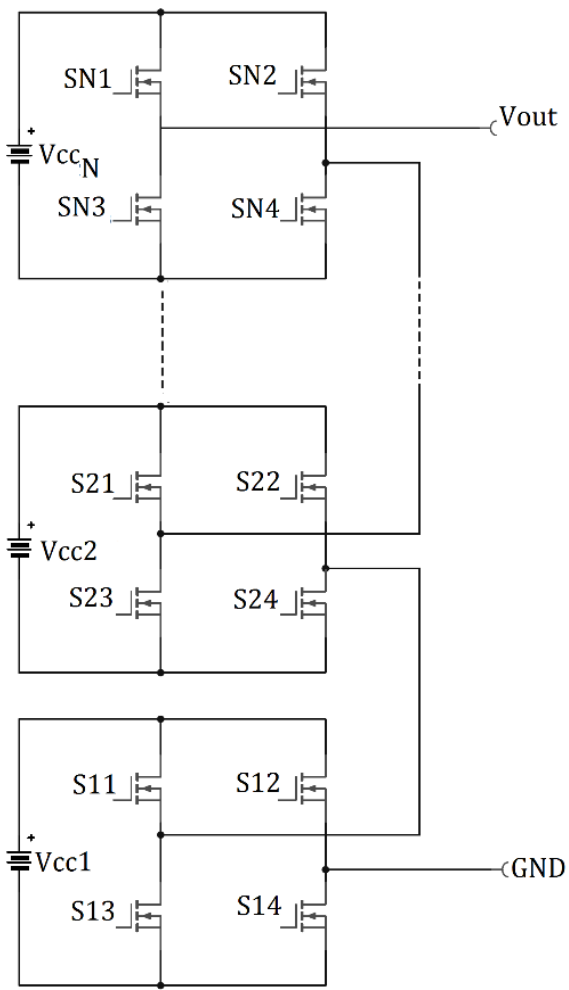

Fig. 3. Inversor multinivel en cascada

En este convertidor cada etapa está compuesta de un arreglo de dispositivos de conmutación en configuración de puente $\mathrm{H}$ y su respectiva fuente de alimentación de DC $\left(V c c_{N}\right)$.

Las etapas se conectan en serie para obtener una forma de onda de salida de múltiples niveles a partir de la selección apropiada del ángulo de disparo de cada transistor. Cuando las fuentes de alimentación de todas las etapas es la misma, a este convertidor se le denomina inversor multinivel en cascada simétrico.

En el caso en que las fuentes de alimentación de las etapas son distintas, a este convertidor se le denomina inversor multinivel en cascada asimétrico. En este último, las fuentes de DC entre las etapas están relacionadas por un factor entero.

\section{MODULACIÓN MCSPWM}

Con el propósito de mejorar el contenido armónico de la señal de salida del convertidor, es posible implementar una modulación sinusoidal de ancho de pulso de múltiple portadora (MCSPWM) (Carrara et al., 1992). En este caso, una señal de referencia sinusoidal es comparada con varias portadoras triangulares en función del número de etapas del convertidor. Las señales obtenidas se emplean para llevar a cabo el disparo de los dispositivos de conmutación de forma apropiada. En la figura 4 se puede apreciar un ejemplo para este tipo de modulación para un convertidor de 9 niveles, mientras que en la figura 5 se muestran las formas de onda moduladas para cada etapa del convertidor. Finalmente, la figura 6 representa la forma de onda del voltaje de salida, que se obtiene a partir de la suma de las salidas de cada etapa. Al modificar los parámetros de las señales, como el índice de modulación, la simetría de la señal triangular o la frecuencia de la misma, se pueden variar las características de la señal de salida del convertidor (tensión RMS, frecuencia de salida, perfil de armónicos).

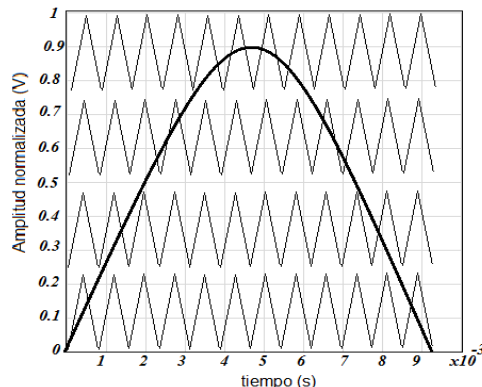

Fig. 4. Modulación MCSPWM de 4 etapas.
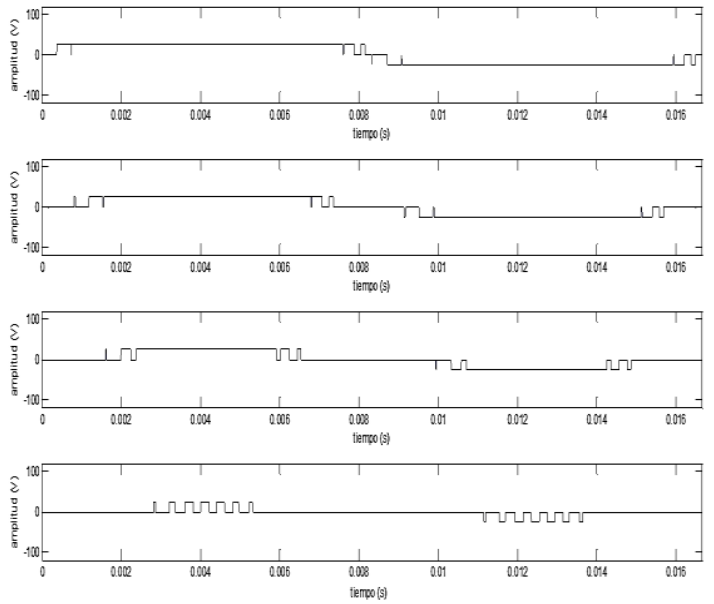

Fig. 5. Formas de onda de salida de cada etapa del convertidor. 


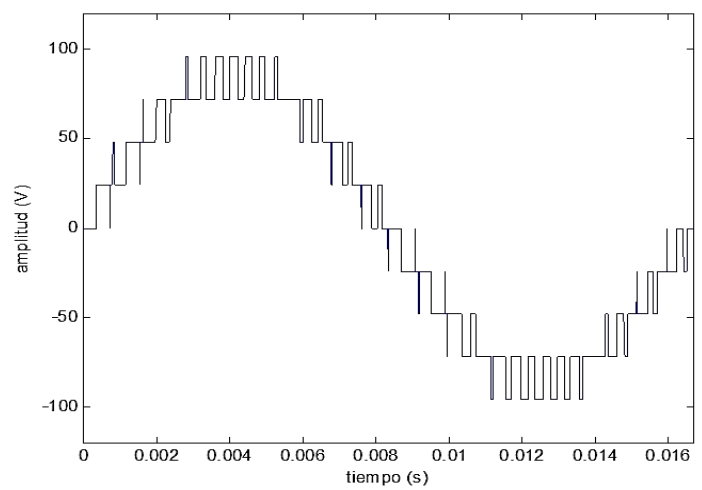

Fig. 6. Forma de onda de salida de 9 niveles.

\section{OPTIMIZACIÓN POR RECOCIDO SIMULADO (SIMULATED ANNEALING)}

El recocido simulado (Simulated Annealing o SA) es un método probabilístico propuesto por Kirkpatrick, Gelett y Vecchi en 1983 (Kirkpatrick, et al., 1983) para encontrar el mínimo global de una función de costo la cual puede tener muchos mínimos locales. El algoritmo SA trabaja imitando el proceso físico por el cual un sólido es enfriado lentamente, hasta que eventualmente su estructura molecular es congelada y este pasa a una configuración de mínima energía.

La versión algorítmica de este proceso inicia con una estimación aleatoria de los valores de las variables de la función de costo. El calentamiento permite modificar aleatoriamente los valores de las variables. Un mayor calor implica mejores variaciones aleatorias. La función de costo retorna la salida $f$, asociada con un conjunto de variables. $\mathrm{Si}$ la salida se decrementa, entonces el nuevo conjunto de variables sustituye al viejo. Si la salida se incrementa, entonces la salida es aceptada, siempre y cuando:

$$
r \leq e^{\left[f\left(P_{\text {old }}\right)-f\left(P_{\text {new }}\right)\right] / T}
$$

Donde $r$ es un número aleatorio y $T$ es una variable análoga a la temperatura. De otra manera, el nuevo conjunto de variables es rechazado. Por lo tanto, incluso si un nuevo conjunto de variables conduce a un costo peor, este puede ser aceptado con una cierta probabilidad. El nuevo conjunto de variables se encuentra tomando un paso aleatorio desde el viejo conjunto de variables:

$$
P_{\text {new }}=d \cdot P_{\text {old }}
$$

Donde la variable d es distribuida uniformemente o normalmente alrededor de $P_{\text {old }}$. Esta variable de control establece el tamaño de paso de modo que, al comienzo del proceso, el algoritmo es forzado a hacer cambios grandes en los valores de las variables. En ocasiones los cambios mueven al algoritmo lejos del óptimo, lo cual fuerza al algoritmo a explorar nuevas regiones del espacio de búsqueda. Luego de un cierto número de iteraciones, los nuevos conjuntos de variables ya no dan lugar a costos más bajos. En este punto los valores de $T$ y $d$ se decrementan por un cierto porcentaje y el algoritmo se repite. El algoritmo se detiene cuando $T=0$. El decremento en $T$ se conoce como programa de enfriamiento. Existen muchas posibilidades para el programa de enfriamiento. Si la temperatura inicial es $T_{0}$ y la temperatura final es $T_{N}$, entonces la temperatura en el paso $n$ está dada por (Hauput y Hautput, 2004):

$$
T_{n}=f\left(T_{0}, T_{N}, N, n\right)
$$

Donde $f$ se decrementa con el tiempo. Algunos programas de enfriamiento son:

- Linealmente decreciente: $T_{n}=T_{0}-n\left(T_{0}-T_{N}\right) / N$.

- Geométricamente decreciente: $T_{n}=0.99 T_{n-1}$

- Hayjek óptimo: $T n=c / \log (1+n)$, donde $c$ es la variación más pequeña requerida para salir de un mínimo local.

Existen muchas otras variaciones. La temperatura usualmente se decrementa lentamente así que el algoritmo tiene chance de encontrar el mínimo correcto antes de intentar ir al punto más bajo en la superficie de búsqueda.

\section{PLANTEAMIENTO DEL PROBLEMA DE OPTIMIZACIÓN}

Como se expuso en la sección 3, la modulación SPWM con múltiple portadora consiste en la comparación de una señal de referencia sinusoidal con una serie de portadoras triangulares. A partir de esta comparación se generan las señales de disparo de las etapas del convertidor. El THD y el voltaje de la componente fundamental dependen en este caso, del índice de modulación $m$, del número de pulsos y de la posición de los pulsos generados. Estas conformarán el conjunto de variables de la función de costo. El índice de modulación es la relación de amplitudes entre la señal de referencia sinusoidal y las portadoras triangulares. El número de pulsos depende de la frecuencia de las señales 
portadoras triangulares y la posición del pulso depende de la forma de la señal triangular. En la figura 7 se muestran tres casos de la forma de onda triangular con los que se obtiene una posición de pulso distinta, variando el tiempo de subida $t r$ y el tiempo de bajada $t f$ de la señal triangular.

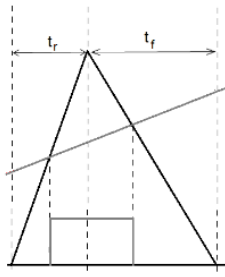

(a)

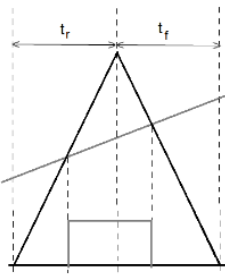

(b)

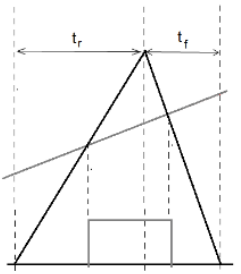

(c)
Fig. 7. Variaciones de la posición de pulso respecto a la disposición de la señal triangularde referencia: $a) t r<t f, b) t r=t f c) t r>t f$.

En MATLAB ${ }^{\circledR}$ es posible generar una forma de onda triangular con la función:

$$
\operatorname{sawtooth}(T, w i d t h)
$$

Donde width es un parámetro escalar entre 0 y 1 , el cual determina la fracción entre 0 y $2 \pi$ en la cual el máximo ocurre. Por ejemplo, si width $=0.5$, la forma de onda obtenida es una triangular simétrica. Si width $<0.5$ entonces $t r<t f$ y si width $>0.5$ entonces $t r>t f$.

El problema de optimización se puede plantear de la siguiente manera:

Minimizar:

$$
T H D[\%]=\left[\frac{1}{a_{1}^{2}} \cdot \sum_{n=3}^{\infty}\left(a_{n}^{2}\right)\right] \cdot 100
$$

Donde:

$a_{1}$ es la amplitud de la armónica fundamental. $a_{n}$ es la amplitud de la $n$-ésima armónica, con $n$ impar.

Sujeto a:

$a_{1}=169.7 \mathrm{Vp}$.

$F_{c} \leq 3 \mathrm{KHz}$

$m \leq 1$.

width $\leq 1$

Donde $a_{1}$ es la amplitud pico de la componente fundamental, $m$ es el índice de modulación, que depende de la amplitud de la señal sinusoidal de referencia, $F c$ es la frecuencia de las señales portadoras triangulares y width corresponde al parámetro de simetría de la señaltriangular.

\section{RESULTADOS}

El esquema de optimización propuesto se simuló en MATLAB para un convertidor multinivel monofásico de 2, 3 y 4 etapas con una amplitud deseada de la componente fundamental de $169.7 \mathrm{~V}$ (120 Vrms), una frecuencia de salida de $60 \mathrm{~Hz}$ y un THD $<5 \%$. Para el cálculo del THD se tuvieron en cuenta los primeros 40 armónicos de la señal de salida.

\subsection{Convertidor multinivel con 2 etapas}

En la figura 8 se observa el conjunto de portadoras triangulares optimizadas junto con la señal de referencia sinusoidal. La figura 9 muestra la forma de onda de salida del convertidor y el perfil de armónicos. En la figura 10 se aprecia la evolución del THD a lo largo de las iteraciones del algoritmo de recocido simulado.

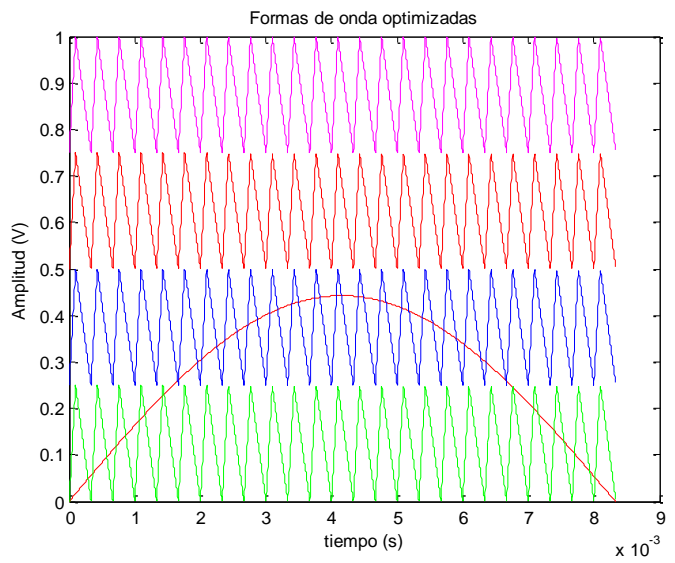

Fig. 8. Señales de referencia y portadora optimizadas para un convertidor de 2 etapas.
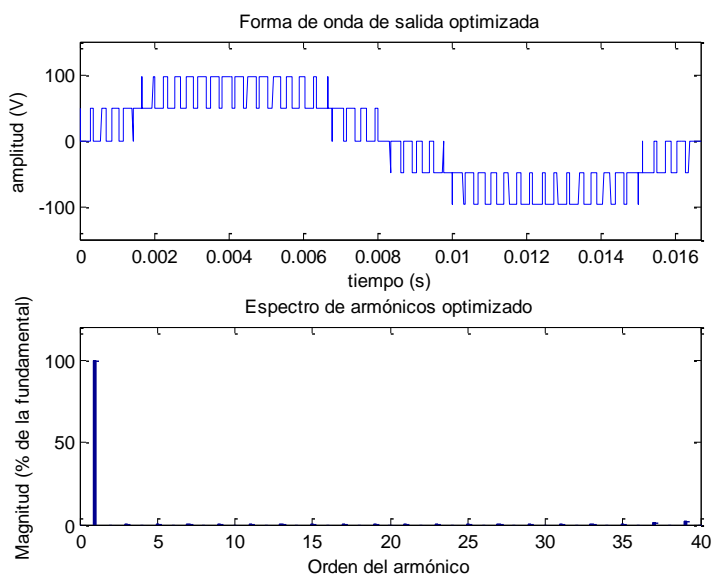

Fig. 9. Forma de onda optimizada y perfil de armónicos para un convertidor de 2 etapas. 


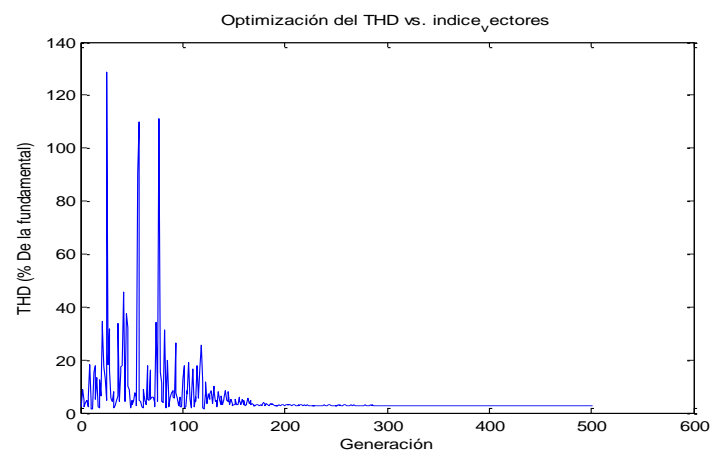

Fig. 10. Evolución del THD del algoritmo SA para un convertidor de 2 etapas.

\subsection{Convertidor multinivel con 3 etapas}

En la figura 11 se observa el conjunto de portadoras triangulares optimizadas junto con la señal de referencia sinusoidal. La figura 12 muestra la forma de onda de salida del convertidor y el perfil de armónicos. En la figura 13 se aprecia la evolución del THD a lo largo de las iteraciones del algoritmo de recocido simulado.

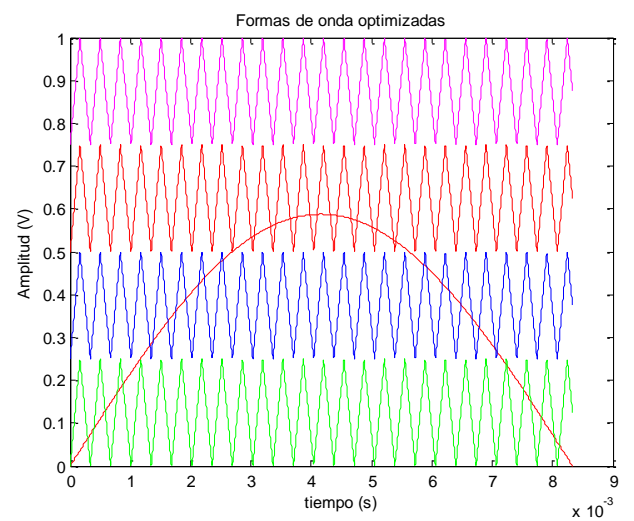

Fig. 11. Señales de referencia y portadora optimizadas para un convertidor de 3 etapas.
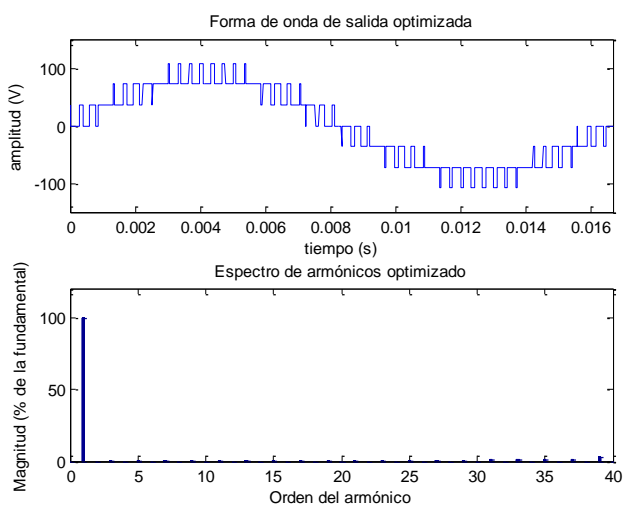

Fig. 12. Forma de onda optimizada y perfil de armónicos para un convertidor de 3 etapas.

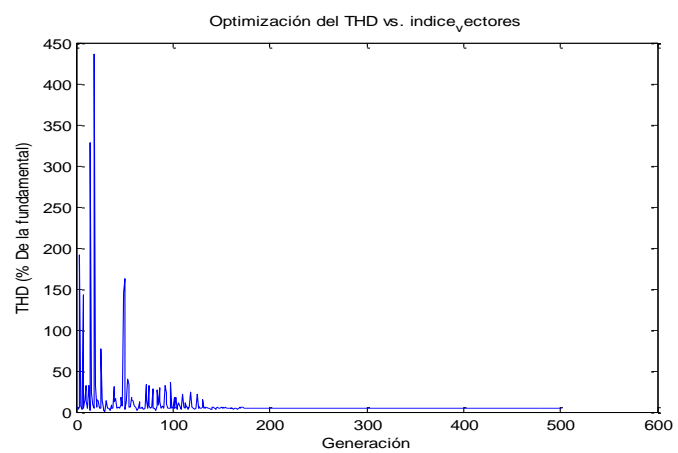

Fig. 13. Evolución del THD del algoritmo SA para un convertidor de 3 etapas.

\subsection{Convertidor multinivel con 4 etapas}

En la figura 14 se observa el conjunto de portadoras triangulares optimizadas junto con la señal de referencia sinusoidal. La figura 15 muestra la forma de onda de salida del convertidor y el perfil de armónicos. En la figura 16 se aprecia la evolución del THD a lo largo de las iteraciones del algoritmo de recocido simulado.

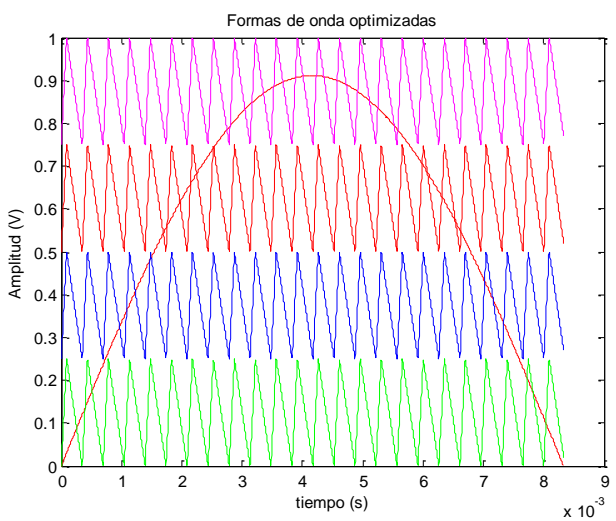

Fig. 14. Señales de referencia y portadora optimizadas para un convertidor de 4 etapas.
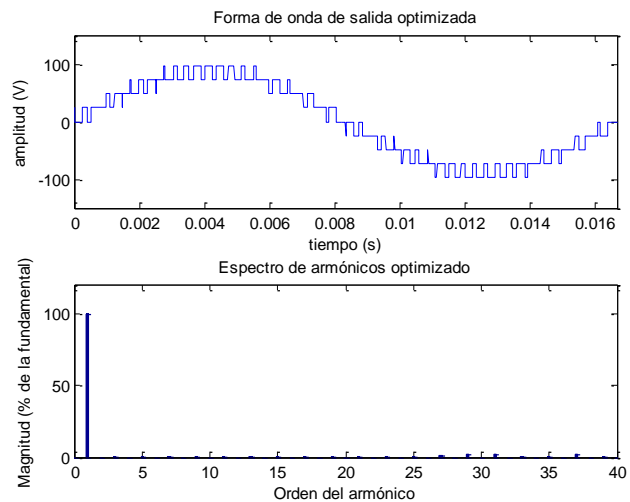

Fig. 15. Forma de onda optimizada y perfil de armónicos para un convertidor de 4 etapas. 


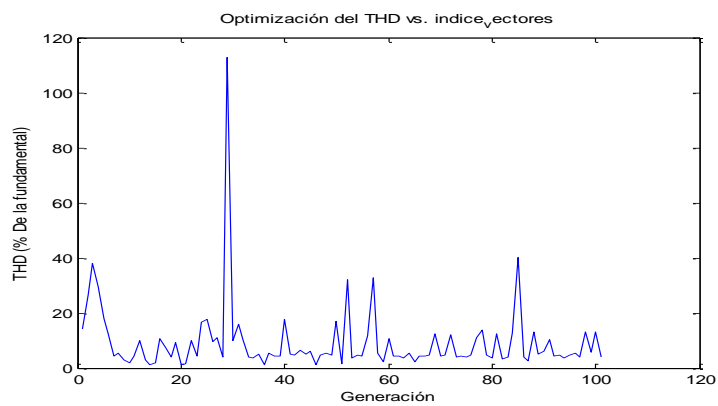

Fig. 16. Evolución del THD del algoritmo SA para un convertidor de 4 etapas.

A continuación se resumen los resultados obtenidos en las simulaciones:

\section{Tabla 1. Resumen de resultados de simulación}

\begin{tabular}{|c|c|c|c|}
\hline $\begin{array}{c}\mathbf{N}^{\boldsymbol{o}} \text { DE } \\
\text { ETAPAS }\end{array}$ & $\begin{array}{c}\mathbf{N}^{\boldsymbol{0}} \text { DE } \\
\text { NIVELES }\end{array}$ & $\begin{array}{c}\text { Vout } \\
\text { (V)MS) }\end{array}$ & THD (\%) \\
\hline 2 & 5 & 174.9 & 4.12 \\
\hline 3 & 7 & 169.25 & 4.02 \\
\hline 4 & 9 & 169.65 & 2.74 \\
\hline
\end{tabular}

\section{CONCLUS IONES}

Como puede observarse en las figuras 8.11 y 14 , el algoritmo de recocido simulado ajusta la forma de onda de las señales de referencia triangular, el índice de modulación y el número de pulsos por semiciclo de forma óptima para satisfacer las restricciones impuestas.

A partir de las figuras 9,12 y 15 se puede concluir que el algoritmo de recocido simulado permite obtener formas de onda de salida con un bajo contenido armónico.

La tabla 1 muestra que las tres configuraciones simuladas permiten satisfacer el requerimiento de un THD por debajo del 5\%, obteniendo en el convertidor de 4 etapas un valor de apenas el $2.74 \%$.

El uso del algoritmo de recocido simulado hace que no sea necesario abordar la solución de una manera formal, ya que el AS explora en el espacio de búsqueda a partir de varios puntos de solución y no se enfoca en encontrar soluciones "exactas" sino la mejor solución al problema.

Como el recocido simulado es un método de optimización heurístico, cada vez que se ejecuta el AS se puede llegar a soluciones ligeramente distintas pero que satisfacen el problema de minimización.

\section{REFERENCIAS}

Skvarenina, T. (2002). "The Power Electronics Handbook". Florida, CRC Press.

Baker, R. y Bennister, L. (1975). "Electric power converter". U.S. Patent 3867643.

Baker, R. (1980). "High-Voltage Converter Circuit". U.S. Patent 04203151.

Lavieville, J. et al. (1997). "Electronic circuit for converting electrical energy and power supply installation making use thereof'. U.S. Patent 5668711.

Meynard, T. et al. (1998). "Electronic circuit for converting electrical energy". U.S. Patent 5706 188.

Rodriguez, J. et al. (2002). "Multilevel inverters: A survey of topologies, controls and applications". IEEE Trans. on Industrial Electronics, Vol. 49, No. 4, pp. 724-738.

Peng, F. y Lai, J. (1997). "Multilevel cascade voltage-source inverter with separate DC sources". U.S. Patent 5642275.

Pandey, A. et al. (2006). "A Review of Multilevel Power Converters". IE Journal-EL.

Malinowski, M. y Styński, S. (2007). "Simulation of single-phase cascade multilevel PWM converters". EUROCON 2007, pp. 1524-1529. Varsovia, 2007.

Rios, F. (2003). "Diseño y construcción de un inversor trifásico multinivel". Tesis de maestría, Universidad Católica de Chile, Santiago de Chile.

Negroni, J. (2007). "Análisis y Diseño de Controladores para Inversores Multinivel en Sistemas Fotovoltaicos Conectados a Red". Tesis Doctoral, Barcelona.

Lega, A. (2007). "Multilevel Converters: Dual Two-Level Inverter Scheme". Tesis Doctoral, Dpto. de Ing. Eléctrica, UB, Italia.

Chavarría, J. (2010). "Diseño e Implementación de un Inversor Multinivel para Sistemas Fotovoltaicos Conectados a Red". Tesis de Maestría, UPB, Barcelona.

Kirkpatrick, S. et al. (1983). "Optimization by Simulated Annealing". Science magazine, Vol. 220, No. 4598, pp. 671-680.

Bertsimas, D. y Tsitsiklis, J. (1993). "Simulated Annealing". Statistical Science, Vol. 8, No. 1, pp. 10-15.

Carrara, G. et al. (1992). "A new multilevel PWM method: a theoretical analysis". IEEE Trans. on Power Elect., vol. 7, pp. 497-505.

Hauput, R. y Hautput, S. (2004). "Practical genetic algorithms", Editorial Wiley-Interscience, $2^{\mathrm{da}}$ edición, Estados Unidos. 